\title{
Enhancing Cell-Edge Performance: A Downlink Dynamic Interference Avoidance Scheme with Inter-Cell Coordination
}

\author{
Mahmudur Rahman, Student Member, IEEE, and Halim Yanikomeroglu, Member, IEEE
}

\begin{abstract}
Interference management has been a key concept for designing future high data-rate wireless systems that are required to employ dense reuse of spectrum. Static or semistatic interference coordination based schemes provide enhanced cell-edge performance but with severe penalty to the overall cell throughput. Furthermore, static resource planning makes these schemes unsuitable for applications in which frequency planning is difficult, such as femtocell networks. In this paper, we present a novel dynamic interference avoidance scheme that makes use of inter-cell coordination in order to prevent excessive inter-cell interference, especially for cell or sector edge users that are most affected by inter-cell interference, with minimal or no impact on the network throughput. The proposed scheme is comprised of a two-level algorithm - one at the base station level and the other at a central controller to which a group of neighboring base stations are connected. Simulation results show that the proposed scheme outperforms the reference schemes, in which either coordination is not employed (reuse of 1) or employed in a static manner (reuse of 3 and fractional frequency reuse), in terms of cell edge throughput with a minimal impact on the network throughput and with some increase in complexity.
\end{abstract}

Index Terms-OFDMA resource allocation, interference avoidance, resource optimization, inter-cell coordination.

\section{INTRODUCTION}

$\mathbf{O}$ RTHOGONAL frequency division multiplexing (OFDM) is being acknowledged as a promising airinterface technology for the next generation wireless local area network such as wireless fidelity (WiFi) [1] to cellular systems like worldwide interoperability for microwave access (WiMAX) [2], 3GPP long term evolution (LTE) of UMTS [3], LTE-Advanced [4], world initiative new radio (WINNER) [5], and WINNER+ [6] systems. Multiuser OFDM or orthogonal frequency division multiple access (OFDMA) is a natural extension to OFDM. Besides its inherent ability to combat inter-symbol interference (ISI) resulting from frequency selective fading, OFDMA offers flexibility in radio resource allocation granularity as each sub-carrier can be allocated, modulated, and coded adaptively to exploit frequency and multi-user diversity gains. In order to meet the high target data-rates in these beyond $3 \mathrm{G}$ cellular systems, dense reuse

Manuscript received February 18, 2009; revised September 5, 2009 and November 8, 2009; accepted December 31, 2009. The associate editor coordinating the review of this paper and approving it for publication was H.-H. Chen.

The authors are with the Department of Systems and Computer Engineering, Carleton University, 1125 Colonel By Drive, Ottawa, Ontario K1S 5B6, Canada (e-mail: \{mmrahman, halim\} @ sce.carleton.ca).

Digital Object Identifier 10.1109/TWC.2010.04.090256 of frequency is required with the obvious pitfall of high intercell interference. Therefore, in order to realize full potential of the OFDMA in a dense reuse environment, employing appropriate interference mitigation technique(s) is essential. To that end, interference mitigation has been identified as one of the major issues currently being investigated by different standardization bodies and forums focusing forthcoming cellular systems.

Interference mitigation techniques are classified into three major categories such as interference cancellation through receiver processing, interference randomization by frequency hopping, and interference avoidance achieved by restrictions imposed in resource usage in terms of resource partitioning and power allocation [2], [7], [8]. The benefits of these techniques are mutually exclusive, and hence, a combination of these approaches is likely to be employed in the system. Our focus in this paper is on interference avoidance, where a dynamic inter-cell interference coordination (ICIC) scheme ${ }^{1}$ that makes use of inter-cell coordination is investigated in a multi-cell environment with aggressive frequency reuse.

Traditionally, inter-cell interference is handled by the classical clustering technique [10], for example, a reuse of 3 . While this technique reduces interference for the cell-edge user terminals (UTs), it compromises system throughput due to resource partitioning. Such partitioning schemes may have been good enough for early networks focusing primarily voice service; however, they are inapplicable to future systems envisioned to support ranges of high data-rate applications, for example, video conference. In the recent years, the fractional frequency reuse (FFR) scheme has attracted the attention of the researchers in different standardization bodies and forums. The motivation behind FFR lies in the fact that UTs in the central area of a cell are more robust against interference due to low path-loss and hence they can tolerate higher reuse compared to those at the cell border suffering from high interference as well as high path-losses. Therefore, it makes sense to use different degrees of reuse factor for UTs in the cell-centre and cell-edge areas. Partial frequency reuse (PFR) [11] and soft frequency reuse (SFR) [12] are two variations of the FFR scheme. A common example of FFR for a network with trisector base stations (BSs) is a blend of reuse factor of 1 and 3 in the cell-centre and cell-edge areas, respectively.

\footnotetext{
${ }^{1}$ The concept and preliminary results are presented in part in IEEE VTC2008-Spring conference [9] and contributed to a WINNER deliverable [7].
} 
Partition of resources into the cell-edge and cell-centre bands determines the effective reuse factor. In most of these schemes, higher power is allocated to the resources used for cell-edge UTs. PFR and SFR schemes are discussed in Section II in greater detail.

Most proposals on FFR rely on static or semi-static coordination among BSs; it is seen in the literature that FFR schemes that use interference coordination in a static or semi-static manner do not provide much gain as cell-edge throughput can only be improved with severe penalty to the system throughput [7]. In addition, such schemes requiring frequency planning cannot be applied to the emerging femtocellular networks [13], as femtocells will be placed at the end user locations in an ad hoc manner making any prior frequency planning difficult. Dynamic coordination schemes, on the other hand, do not require prior frequency planning and operate based on dynamic interference information from surrounding transmitters. Therefore, dynamic schemes are not only effective to avoid interference in macrocell-macrocell scenario, they are also capable of handling interference from macrocells if applied to femtocell BSs. Dynamic inter-cell coordination based schemes can best exploit channel variations to achieve maximum interference avoidance gain; however, only a few such studies can be found in the literature [9], [14]-[16]. In [14], a dynamic inter-cell coordination scheme is studied in a simplistic scenario and assumptions, where scheme chooses a reuse pattern from four defined patterns with varying degrees of partitioning (e.g., reuse of 1 to reuse of 3). The dynamic FFR scheme, studied in [15], partitions resources dynamically into super-group and regular-group which are allocated to cells and sectors, respectively. The scheme achieves higher system throughput, however, the cell-edge performance degrades as compared to a static FFR scheme. A distributed dynamic interference coordination scheme in the context of LTE system is studied in [16]. Interference coordination is studied using interference graph approach in [17] and [18].

In this paper, we extend our previous work presented in [9] as follows. The heuristics to prepare utility matrix have been modified, where two different threshold functions are introduced to provide different degrees of trade-off between throughput and cell-edge performance. An additional scenario in which restricted chunks can also be used with lower transmit power is considered. In addition to the full-buffer traffic assumption, we also test our algorithm with a more realistic traffic model according to Poisson arrival. The reference Reuse 3 and PFR schemes are included in addition to Reuse 1 to which the performance of our schemes is compared. Finally, we have employed proportional fair (PF) scheduler as well as the iterative Hungarian scheduler used in [9].

A large number of available literature on resource allocation in OFDMA concentrate primarily on various optimization techniques in a single cell context [19]-[21]. However, in designing practical networks, optimization should be performed in a multi-cell environment taking into account one of the most important performance limiting factors, inter-cell interference. To the authors' knowledge, resource optimization in a multicell environment has not yet been investigated well and only a few research works on multi-cell allocation can be found in the literature [22], [23]. In [22], a linear programming (LP) formulation is proposed, where sub-channels are partitioned and assigned fixed reuse factors such that user terminals (UTs) at the cell edge can only use sub-channels with higher reuse factors. From the optimization point of view, radio resource allocation with resource restriction in a multi-cell environment can be seen as a three-dimensional assignment problem; it should determine which part of the resources should be restricted in which cell as well as which available resources will be allocated to which set of UTs. Three dimensional assignment problem is NP-hard [24], and hence, a good sub-optimal solution with reasonable complexity is desirable. Contrary to the scheme in [22] and other static FFR schemes, our proposed scheme does not require any prior resource partitioning or cell planning. Such proactive partitioning of resources in a static manner reduces resource utilization. On the other hand, our proposed scheme results in a dynamic and efficient reuse factor for each sub-channel depending on mutual interference situations and the UTs' service status.

In this paper, a chunk is defined as a collection of consecutive sub-carriers over a defined time period, which is regarded as the minimum granularity of the radio resource allocation unit (analogous to physical resource block (PRB) used in 3GPP LTE and LTE-advanced studies). By using intercell coordination, our proposed scheme thrives to enhance throughput on allocated chunks considering the service status of the cell edge UTs. In particular, any optimal or sub-optimal allocation scheme, such as in [23], can exploit multiuser diversity to achieve maximized sector throughput by optimally or sub-optimally assigning best chunks to UTs. However, as the cell edge UTs experience higher path-losses on the desired links and higher interference from nearby cells, these scheduling schemes tend to overlook such disadvantaged UTs. Therefore, interference avoidance is crucial in order to provide enhanced data rate to the cell edge UTs.

The proposed scheme is comprised of two separate algorithms residing at the BS and at a central entity. Based on the interference received by its UTs and their service status, each sector (via its BS) sends a request to the central controller; this request incorporates a tentative list of chunks to be restricted at the surrounding dominant interferer sectors. This request also includes the utility measure of the chunks in the requesting sector. The central controller gathers all such requests and processes to prepare a refined list of chunk restrictions to be applied in all involved sectors in different cells. The central controller sends the restriction decision to all involved sectors. This restriction process is refreshed from time-to-time within an interval which is shorter than the channel coherence time. Scheduler takes the restriction decision into consideration. In order to achieve two-tier benefits, this approach of network layer resource coordination for interference avoidance can be complemented by physical layer processing defined as coordinated multi-point (CoMP) transmission/reception [25] in LTE-Advanced systems. We have considered two different scenarios - restricted chunks are not used at all in the first scheme and chunks are used only with reduced power in the second scheme. Details of the proposed schemes are presented in a later section.

The performance of the proposed schemes is compared with that of some reference schemes, for example, reuse of 1 , reuse 
of 3, and the PFR schemes. Reuse 1 scheme represents the no coordination case, and the other two represent cases where coordination is used in a static manner.

The remainder of this paper is organized as follows. We provide a summary of efforts on interference avoidance in the context of different standardization activities and forums in Section II. The system model is described in Section III. Details of the proposed scheme are presented in Section V. Section VI describes system and simulation parameters. Numerical results, obtained through extensive simulations, are discussed in Section VII. Section VIII addresses the implementation complexity issues of the proposed schemes followed by the conclusions in Section IX.

\section{Partition-Based Static Coordination Schemes}

If the available frequency spectrum is reused in each sector without imposing any restriction to frequency resource usage or power allocation, it achieves a reuse factor of 1, i.e., the worst inter-cell ${ }^{2}$ interference situation.

On the other hand, if the available frequency spectrum is divided into three sub-bands and each sector is given a sub-band which is orthogonal to neighboring sectors' sub-bands, then a reuse of 3 can be achieved. This clustering obviously gives improved inter-cell interference, however, with a significant resource restriction loss due to partitioning. Fig.s 1.a \& 1.b show reuse 1 and 3 schemes, respectively, where total transmit power per sector transmitter remains constant in both cases. While reuse 1 does not employ any interference coordination, reuse 3 can be regarded as an extreme case of partition-based static interference coordination. The FFR schemes achieve an effective reuse factor between 1 and 3. Two variations of FFR schemes, namely SFR and FFR, are shown in Fig.s 1.c \& 1.d and are elaborated below.

\section{A. Soft Frequency Reuse (SFR)}

SFR, a variation of FFR, employs zone-based reuse factors in the cell-centre and the cell-edge areas. Restrictions are imposed in terms of allocation of frequency and power in the zones. The term soft reuse comes from the fact that effective reuse of the scheme can be adjusted by the division of powers between the frequencies used in the centre and edge bands. In order to provide enhanced services to disadvantaged UTs near the cell boundary, SFR was proposed in [12] and [26] within 3GPP LTE framework.

For 3-sector cell sites, as shown in Fig. 1.c, the cell-edge band (also termed as major band) uses $1 / 3$ of the available spectrum which is orthogonal to those in the neighboring cells and forms a structure of cluster size of 3 . The cell-centre band (also called minor band) in any sector is composed of the frequencies used in the outer zone of neighboring sectors. Each group is assigned transmission power depending on the desired effective reuse factor while keeping the total transmission power fixed. Higher transmit power is used on the major band as shown in the right side of Fig. 1.c. Assume that the power per chunk is $P_{t} / N$ in the case of reuse factor

\footnotetext{
${ }^{2}$ The terms "sector" and "cell" are used interchangeably in this paper, and therefore, inter-cell interference refers to interference received by a UT in a sector from any other sector transmitter using same frequency.
}

of 1 without coordination (as shown in Fig. 1.a) and power per chunk used in the cell-edge (major) band is $\alpha_{p} P_{t} / N$ of the SFR scheme. Here, $P_{t}$ is the total transmit power per sector, $N$ is the number of chunks, and $\alpha_{p}$ is a power amplification factor whose value is greater than 1. As the total transmit power is constant, the power per chunk in the minor band of SFR would have to be $P_{t}\left(3-\alpha_{p}\right) / 2 N$ giving a ratio of powers of minor to major bands as $\left(3-\alpha_{p}\right) / 2 \alpha_{p}$.

The major band can be used in the cell-centre as well if it is not occupied by the cell-egde UTs, but the minor band is available to the centre area only. Due to this scheduling restriction, adjusting the power ratio from 0 to 1 effectively moves the reuse factor from 3 to 1 . Therefore, SFR is seen as a compromise between reuse 1 and 3 in a network with trisector BSs. UTs are categorized into cell-edge and cell-centre based on user geometry determined by the received signal power (averaged over multipath fading) taking into account the large-scale path-loss, shadowing, and antenna gains.

\section{B. Partial Frequency Reuse (PFR)}

Contrary to SFR, the idea of the partial frequency reuse (PFR) is to restrict portion of the resources so that some frequencies are not used in some sectors at all. This concept was first presented in [27]. The effective reuse factor of this scheme depends on the fraction of unused frequency. The PFR and some of its variants are studied in the 3GPP and WINNER projects (see, for example, [11] and [7]). An example of PFR for sites with 3 sectors is shown in Fig. 1.d. Let us assume that the available system bandwidth is $\beta$ which is divided into inner and outer zones with $\beta_{i}$ and $\beta_{o}$, respectively. Band $\beta_{i}$ is used with a reuse factor of 1 , and for the tri-sector BSs, the reuse factor for $\beta_{o}$ is usually 3 in the outer zone. In this case, the effective frequency reuse factor is given by $\beta /\left(\beta_{i}+\left(\beta_{o} / 3\right)\right)$. Therefore, the effective reuse of PFR scheme is always greater than 1. Similar to SFR scheme, the power used on frequencies in the outer zone can be amplified as shown in Fig. 1.d.

\section{SYSTEM MODEL}

Let us consider a network layout with 19 BS sites each with 3 hexagonal sectors as shown in Fig. 2. Sectors are equipped with $120^{\circ}$ directional transmit antennas, while the UTs' receive antennas are considered to be omni-directional. The antenna gain pattern for the transmit antenna is provided in Section VI. This layout represents the baseline simulation test scenario in most studies relating to LTE, WiMAX, and WINNER [28].

We assume that the system uses cell-specific orthogonal reference signals [3]; UTs know the reference signals of neighboring first-tier sectors and they are able to determine interference separately. It is evident that for a downlink transmission to a UT in any sector, one of its first-tier sectors is likely to be the most dominant interferer. Let us consider Fig. 2 as an example; due to relative locations and antenna directivity, a cell-edge UT in sector 1 of BS1 may receive the most dominant interference from sector 2 or 3 of BS1 (depending on the UT location), or from sector 2 or 3 of BS2, or from sector 3 of BS3, or from sector 2 of BS7. A cell edge UT experiences higher path-loss and receives 


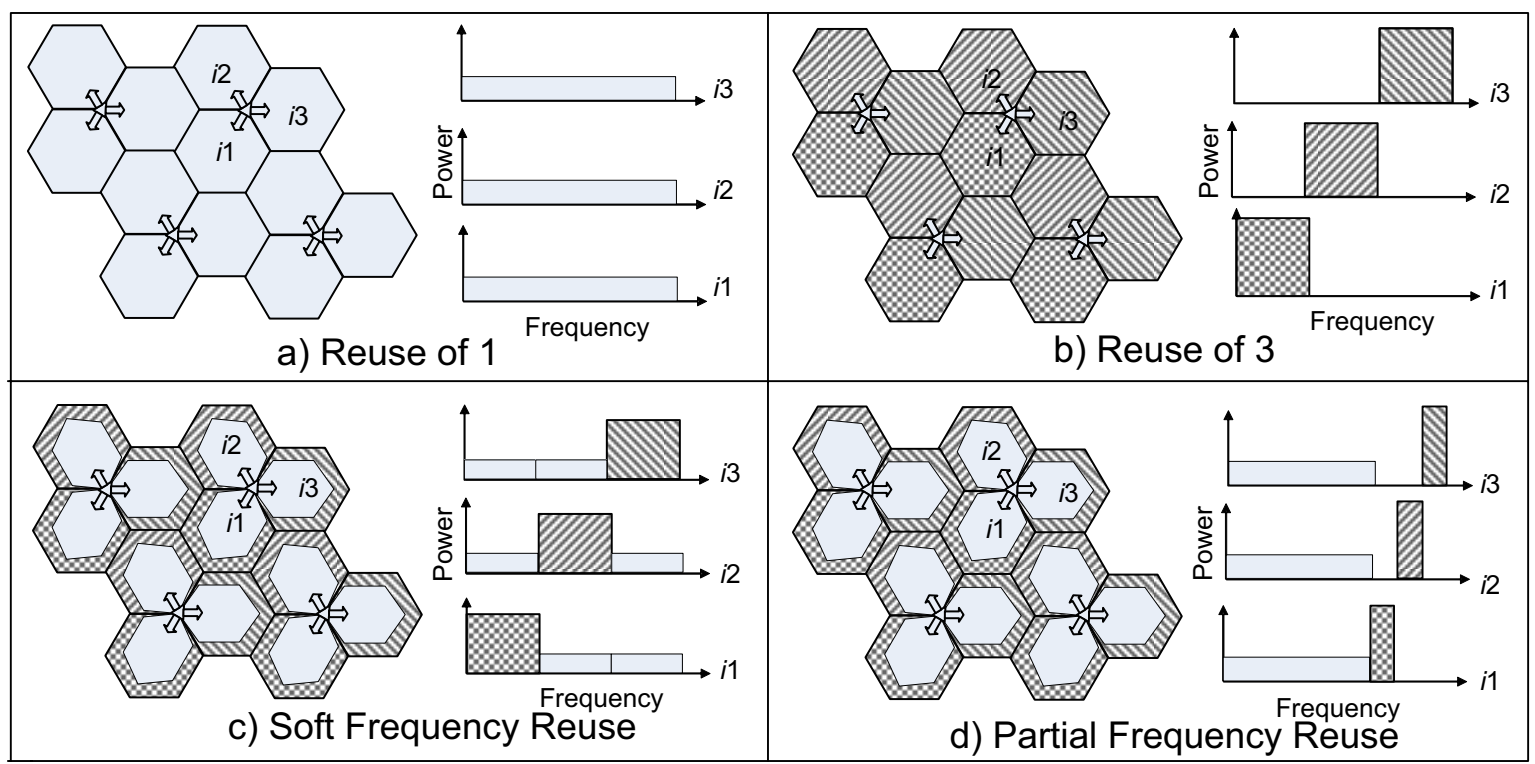

Fig. 1. Reference schemes.

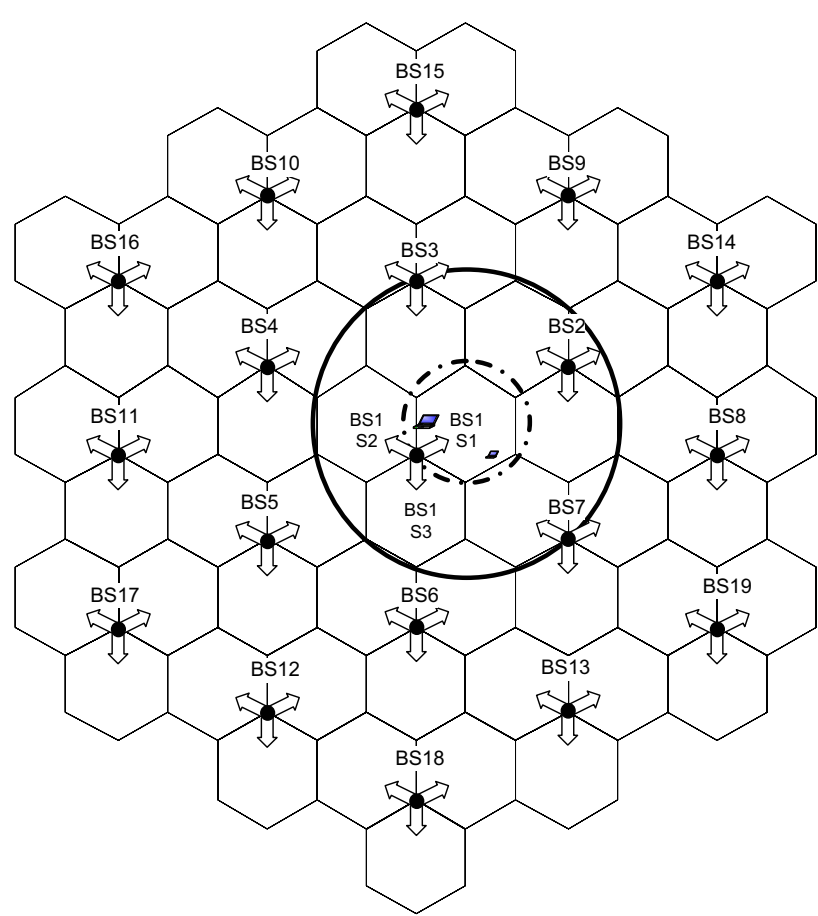

Fig. 2. Network layout under investigation.

significant interference from the sectors of the nearby cells. Also, UTs closer to the serving sector may experience severe interference from the neighboring sectors of own cell. As a consequence, these UTs are susceptible to see more poorquality chunks having low signal-to-interference plus noise ratios (SINRs). Any optimal or sub-optimal allocation scheme that aims to maximize network throughput may overlook such disadvantaged UTs as they are less attractive to contribute to the total throughput compared to those closer to the BS. Therefore, it is very important to avoid interference on such UTs in order to guarantee their minimum required rates. For
TABLE I

LIST OF SYMBOLS USED

\begin{tabular}{|c|c|}
\hline$j$ & index of the first-tier dominant interfering sectors \\
\hline$k$ & index of non-dominant interfering sectors \\
\hline$m$ & UT index \\
\hline$n$ & chunk index \\
\hline$M$ & number of UTs per sector \\
\hline$N$ & number of available chunks per sector \\
\hline$P_{t}$ & total power per sector \\
\hline$P_{c}$ & power per chunk \\
\hline$P_{N}$ & average thermal noise power \\
\hline$H_{m, n}$ & channel gain seen by UT $m$ on chunk $n$ \\
\hline$\gamma_{m, n}$ & SINR experienced by UT $m$ on chunk $n$ \\
\hline$r_{m, n}$ & achievable rate on chunk $n$ for UT $m$ \\
\hline$u_{m, n}$ & utility of chunk $n$ for UT $m$ \\
\hline$U_{M \times N}$ & utility matrix for all chunks and all UTs \\
\hline $\begin{array}{l}\rho_{m, n} \\
\Psi\end{array}$ & $\begin{array}{l}\text { indicator showing allocation of chunk } n \text { to UT } m \\
\text { set of dominant interferers sorted in descending order }\end{array}$ \\
\hline$d_{m}$ & UT demand factor \\
\hline$I_{n}$ & indicator to show whether chunk $n$ is restricted or not \\
\hline$R_{m}$ & time average throughput achieved by UT $m$ \\
\hline $\bar{R}$ & average throughput across all UTs \\
\hline$r_{m}^{T H}$ & $m^{t h}$ UT's rate threshold \\
\hline
\end{tabular}

convenience, we list the symbols used in this section and onward in Table I. To determine chunk restrictions optimally, we formulate a utility maximization problem as follows:

maximize

$$
\sum_{i}\left[\sum_{m=1}^{M} \sum_{n=1}^{N} u_{m, n}^{(i)} \rho_{m, n}^{(i)}\right] ; \quad u_{m, n}^{(i)}=r_{m, n}^{(i)} d_{m}^{(i)},
$$

subject to

$$
\rho_{m, n}^{(i)} \in\{0,1\} ; \forall\{m, n\},
$$

$$
I_{n}^{(i)}=\sum_{m=1}^{M} \rho_{m, n}^{(i)}= \begin{cases}0 ; & \text { chunk } n \text { is restricted in } i \\ 1 ; & \text { otherwise, }\end{cases}
$$

where $u_{m, n}^{(i)}$ and $r_{m, n}^{(i)}$ are the achievable utility and the rate (in bps/Hz) on chunk $n$, respectively, seen by UT $m$ in sector $i$; 
$d_{m}^{(i)}$ is the UT demand factor that indicates the service status of UT $m$. We define $d_{m}^{(i)}$ as follows: $d_{m}^{(i)}=\bar{R}^{(i)} /\left(R_{m}^{(i)}+\delta\right)$, where $R_{m}^{(i)}$ is the average throughput of UT $m$ over a certain timewindow, and $\bar{R}^{(i)}$ is the average throughput across all UTs and is given by $\bar{R}^{(i)}=\left(\sum_{m=1}^{M} R_{m}^{(i)}\right) / M$. A rate deprived UT, such as one near the cell-edge, will have a higher demand factor. Therefore, the utility provides advantages to rate deprived UTs to boost their performance. The parameter $\delta$ has a small value that prevents $d_{m}^{(i)}$ from being $\infty$. Constraints in (2) indicate that the problem is binary integer type, where $\rho_{m, n}$ is 1 if the $n^{\text {th }}$ chunk is used for UT $m$ and it is 0 otherwise. The indicator in constraint (3) indicates whether a chunk is restricted in sector $i$ or not; also, if a chunk is not restricted in $i$, it can only be assigned to one UT.

The SINR, $\gamma_{m, n}^{(i)}$, and resulting achievable rate, $r_{m, n}^{(i)}$, depend largely on interference received from the neighboring first-tier sectors. Let us denote $j$ and $k$ as the indices of the first-tier dominant and other non-dominant distant inter-cell interferers, respectively, for the UTs in sector $i$. For chunk $n$, the SINR seen at UT $m$ in sector $i$ can be expressed by,

$$
\gamma_{m, n}^{(i)}=\frac{P_{c} H_{m, n}^{(i, i)}}{P_{c} \sum_{k=1}^{K} H_{m, n}^{(i, k)} \cdot I_{n}^{(k)}+P_{c} \sum_{j=1}^{J} H_{m, n}^{(i, j)} \cdot I_{n}^{(j)}+P_{N}},
$$

where $P_{c}$ is the transmit power applied on each chunk derived from equal power distribution; that is, $P_{c}=P_{t} / N$, where $P_{t}$ is the total transmit power per sector and $N$ is the number of available chunks. Parameter $P_{N}$ is the thermal noise power over the chunk bandwidth. Parameters $H_{m, n}^{(i, k)}$ and $H_{m, n}^{(i, j)}$ are the link gains to the first-tier dominant and other non-dominant interferer sectors, respectively. The parameter $H$ includes large scale path-loss, antenna gains and directivity, fading, and shadowing. The indicators $I_{n}^{(k)}=\sum_{m=1}^{M} \rho_{m, n}^{(k)}$ and $I_{n}^{(j)}=\sum_{m=1}^{M} \rho_{m, n}^{(j)}$ take the value of 0 or 1 depending on whether or not the $n^{\text {th }}$ chunk is restricted in dominant sector $k$ and non-dominant sector $j$, respectively. Obviously, the first summation in the denominator of (4) has the dominant effect on the quality of the chunk, i.e., most interference avoidance gain can be achieved by imposing restrictions on these interferers.

We express $\gamma_{m, n \mid \Psi}^{(i)}$ as the SINR on chunk $n$ at UT $m$ given a set of sorted dominant interferers, $\Psi$, are not allowed to use chunk $n$. The vector $\Psi$ is sorted by the descending order of interference powers which varies from $\Psi=\{\}$ to $\Psi=\left\{\psi_{1}, \psi_{2}, \cdots, \psi_{K}\right\}$, representing no interferer restriction to all first-tier interferer restriction. Therefore, it is evident that $\gamma_{m, n \mid \Psi=\{\}}^{(i)}<\gamma_{m, n \mid \Psi=\left\{\psi_{1}\right\}}^{(i)}<\cdots<\gamma_{m, n \mid \Psi=\left\{\psi_{1}, \psi_{2}, \cdots, \psi_{K}\right\}}^{(i)}$ representing the SINRs when none, one, two, and so on dominant interferers are restricted to use chunk $n$ in their sector(s). The restriction can be either in the form that restricted chunks are not used at all (as in (1)-(4)) or used only with reduced transmit power.

For a target bit error rate (BER), modulation and coding scheme, the above SINRs can be mapped to achievable rates as $r_{m, n \mid \Psi=\{\}}^{(i)}<r_{m, n \mid \Psi=\left\{\psi_{1}\right\}}^{(i)}<\cdots<r_{m, n \mid \Psi=\left\{\psi_{1}, \psi_{2}, \cdots, \psi_{K}\right\}}^{(i)}$. Clearly, the minimum and maximum rates can be achieved as $r_{m, n \mid \Psi=\{\}}^{(i)}$ and $r_{m, n \mid \Psi=\left\{\psi_{1}, \psi_{2}, \cdots, \psi_{K}\right\}}^{(i)}$, respectively; however, the incremental gain from interferer restrictions diminishes as more and more interferers are restricted, because $\Psi$ is arranged according to the descending order of interference powers.

Solving the interference coordination problem networkwide, as stated in (1)-(4), is computationally prohibitive. Therefore, we resort to a heuristic approach, where each sector locally finds a set of chunks to be restricted, in the form of a wish-list, in each surrounding first-tier dominant interferer sector. This list is prepared based on the inter-cell interference and UTs' demand factors. A utility matrix, $U_{M \times N}$, is prepared using heuristics and the Hungarian algorithm (also known as Kuhn-Munkres algorithm) [29] is applied to it in order to find tentative chunk-to-UT preferences and resulting restriction wish-list. However, as every sector is a dominant interferer to UTs in some other sectors, it is likely that there will be conflicts in restriction requests. Therefore, a physical or a logical central entity is required to resolve the conflicting requests optimally. This central (or localized central) controller can be a node functionally similar to a radio network controller (RNC) in 3G systems or a mobility management entity (MME) in LTE, or any logical entity residing within a physical node such as an evolved NodeB (eNB) with required connectivity and processing capability. The eNBs having central processing capability can be strategically and sparsely placed in the coverage area. These eNBs can communicate with other regular eNBs using X2 interface.

The proposed inter-cell interference coordination scheme is comprised of two separate algorithms; one is located at the BS level that prepares the chunk restriction requests and the other resides at the central controller that resolves restriction request conflicts. The working principle of the scheme can be summarized as below:

- UTs send channel state information (CSI), including information on two most dominant interference received from their first-tier sectors, to the serving sector.

- Each sector prepares a utility matrix based on the channel states and UTs' demand factors.

- Each sector iteratively applies Hungarian algorithm to the utility matrix to find chunk restriction requests for each of its dominant interferer neighbors.

- Each sector forwards restriction request list to the central entity.

- The central entity processes requests from all involved sectors and resolve conflicting requests based on the utility values in an optimal manner.

- The central entity then forwards each sector a decided set of chunks that are to be restricted by its scheduler.

The list of restricted chunks remains valid over a long enough time-period but shorter than the channel coherence time. In the following section, we first discuss the effects of scheduling and dominant interference on throughput and fairness. Then, we present our algorithms elaborately in the subsequent section.

\section{EFFEcts of Scheduling ANd Dominant INTERFERENCE ON THROUGHPUT AND FAIRNESS}

Scheduling has a profound effect on the throughput and fairness that can be achieved with a given amount of resources. 


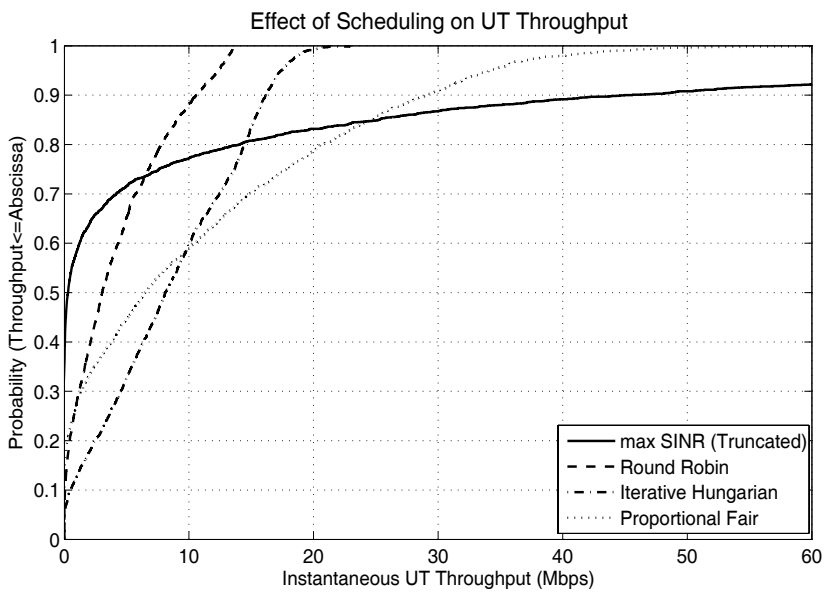

Fig. 3. Effect of scheduling on UT throughput.

To show the effect of scheduling on UT throughput, we provide Fig. 3, where different schedulers such as maximum SINR, round-robin, iterative Hungarian, and proportional fair are used. For the sake of organization, we defer the details of the simulation parameters until Section VI. While maximum SINR scheduling can achieve the highest sector throughput, it severely punishes the cell-edge UTs (lower tail of the CDF). Round-robin scheduler can provide better service to edge UTs, however, it may result in poor sector throughput. The iterative Hungarian and proportional fair schedulers can be seen as schemes that exhibit good compromise between cell-edge and sector throughput. Hungarian algorithm is an efficient, channel aware, and low complexity solution to integer linear programming for one-to-one assignment problem. However, if a UT needs multiple chunks in order for it to achieve the required rate, the iterative Hungarian method rather becomes a good sub-optimal solution. The proportional fair is also a channel aware scheduler, where scheduling priorities are determined by the filtered average UT throughput updated over a certain time window.

In order to have a glimpse of the effect of dominant interference, we use simulations and observed the UT throughput in a sector with different number of interferers restricted. Fig. 4 shows the CDF of UT throughput when none, one, and two dominant interferers are restricted. Here, full-buffer traffic model and iterative Hungarian scheduler have been considered. It is seen from the figure that most avoidance gain can be achieved by restricting the most dominant interferer and the gain is insignificant beyond $\Psi=\left\{\psi_{1}, \psi_{2}\right\}$ ( $\Psi=\left\{\psi_{1}, \psi_{2}, \psi_{3}\right\}$ and onward are not shown in the figure). The inset of Fig. 4 shows the percentage of chunks restricted for $\Psi=\left\{\psi_{1}\right\}$ and $\Psi=\left\{\psi_{1}, \psi_{2}\right\}$. Note that for each chunk, interferers are restricted only when there is a rate gain from restriction. For example, if $r_{m, n \mid \Psi=\left\{\psi_{1}\right\}}^{(i)}<r_{m, n \mid \Psi=\left\{\psi_{1}, \psi_{2}\right\}}^{(i)}$, then $\Psi=\left\{\psi_{1}, \psi_{2}\right\}$, else $\Psi=\left\{\psi_{1}\right\}$ is used. It can also be observed from the figure that on average around $12.5 \%$ and $20 \%$ of chunks are to be restricted in each surrounding sector in order to obtain the throughput gain observed in the figure. If a sector restricts these many chunks in its surrounding sector, it will also receive similar chunk restrictions on average from each of its neighbors causing a large number of chunk

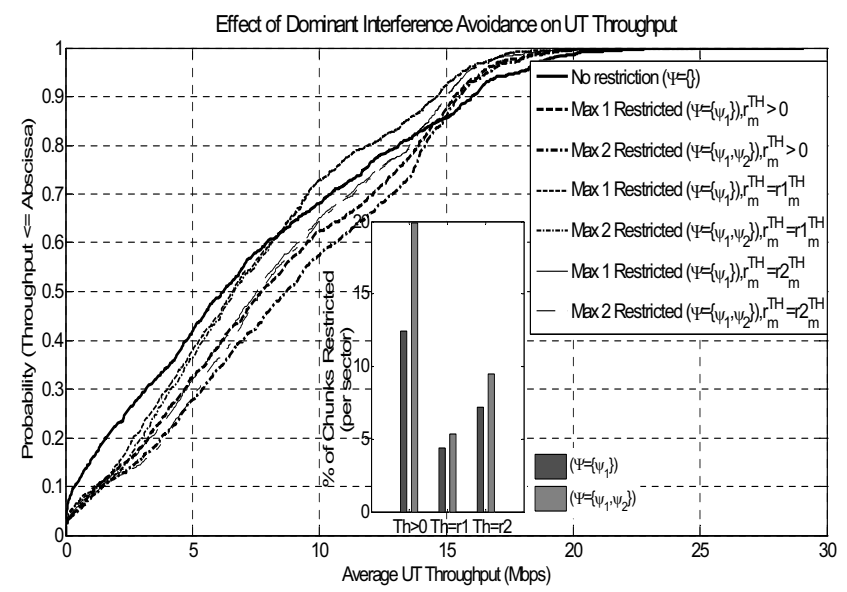

Fig. 4. Effect of dominant interferer restrictions on UT throughput.

restrictions in each sector and resource loss as a consequence. For simplicity, Fig. 4 does not consider the resource loss due to restrictions. Detailed results considering resource loss are presented in Section VII. It is evident that in order to limit the number of restrictions and hence resource loss, only justifiable restrictions should be made. To that end, we present two cases when chunk restrictions are made only when significant gains can be obtained considering the service of the UTs. A sector decides on restrictions based on a threshold value $r_{m}^{T H}$ given as follows:

$$
r 1_{m}^{T H}= \begin{cases}e^{\left(1-d_{m}\right)} ; & d_{m} \geq 1 \\ \infty ; & d_{m}<1\end{cases}
$$

and

$$
r 2_{m}^{T H}=e^{\left(1-d_{m}^{2}\right)} .
$$

In (5) and (6), restrictions are made considering the UTs' current service status. In the former, restrictions are made only in favor of those that have received less than the average service in the sector, while restrictions are made also for the UTs with good service status in the latter, given only when a considerable gain can be achieved. That is, if $r_{m, n \mid \Psi=\left\{\psi_{1}\right\}}^{(i)} \geq r_{m, n \mid \Psi=\{\}}^{(i)}+r_{m}^{T H}$, then the most dominant interferer will be restricted to favor UT $m$ and if $r_{m, n \mid \Psi=\left\{\psi_{1}, \psi_{2}\right\}}^{(i)} \geq r_{m, n \mid \Psi=\left\{\psi_{1}\right\}}^{(i)}+r_{m}^{T H}$, then the two most dominant interferers can be restricted. In (6), the threshold function is considered steeper in order to impose the fact that restrictions are made in favor of UTs with good service rate only when significant gains are foreseen. The throughput plots for the threshold criteria in (5) and (6) are shown in Fig. 4. The following important observations can be drawn by analyzing Fig. 4:

- Higher throughput can be achieved by restricting more and more chunks which implies more penalties to neighboring sectors.

- Substantial gain can be achieved by restricting only the most dominant interferer. Attempts should be made to restrict only this interferer, with the exception for severely rate deprived UTs for which the two most dominant interferers can be restricted.

- The number of restricted chunks and performance can be tuned by choosing an appropriate gain threshold value. 
- The performance using the threshold function given by (6) is superior compared to that in (5) with slight increase in the number of restricted chunks. We use both threshold functions to derive detailed performance results as presented in Section VII.

\section{Interference Avoidance Algorithms}

In this section, we describe the sector-level and the central algorithms as follows. Sector-level algorithm prepares utility matrix and prepares chunk restriction requests for each of its first-tier interferers. The central algorithm resolves any conflicting restriction requests and prepares final restriction list for each sector.

\section{A. Sector-Level Algorithm}

Two major functions are associated with the sector-level algorithm- namely, 1) preparation of a utility matrix using the threshold-based restrictions derived from the channel conditions and UT demands, and 2) Preparation of restriction requests from the tentative chunk-to-UT allocation by using iterative Hungarian algorithm. The pseudo-codes for the sector level algorithm are presented in Table II.

1) Preparation of the utility matrix: In order to construct the utility matrix in sector $i$, the following steps are repeated for each UT $m$ and each chunk $n$.

- Dominant interferers are sorted in descending order of interference powers into a dominant interferer set.

- The conditional SINRs of chunk $n$ for UT $m$, i.e., $\gamma_{m, n \mid \Psi=\{\}}^{(i)}, \quad \gamma_{m, n \mid \Psi=\left\{\psi_{1}\right\}}^{(i)}$, and $\gamma_{m, n \mid \Psi=\left\{\psi_{1}, \psi_{2}\right\}}^{(i)}$, which correspond to restrictions of none, one, and two most dominant interferers, respectively, are calculated from the SINR expression in (4).

- The achievable rates for the above SINRs, i.e., $r_{m, n \mid \Psi=\{\}}^{(i)}, r_{m, n \mid \Psi=\left\{\psi_{1}\right\}}^{(i)}$, and $r_{m, n \mid \Psi=\left\{\psi_{1}, \psi_{2}\right\}}^{(i)}$ are determined.

- If $r_{m, n \mid \Psi=\left\{\psi_{1}\right\}}^{(i)} \geq r_{m, n \mid \Psi=\{\}}^{(i)}+r_{m}^{T H}$, sector $\psi_{1}$ is marked to be restricted if chunk $n$ is to be assigned to UT $m$.

- If $r_{m, n \mid \Psi=\left\{\psi_{1}, \psi_{2}\right\}}^{(i)} \geq r_{m, n \mid \Psi=\left\{\psi_{1}\right\}}^{(i)}+r_{m}^{T H}$, sector $\psi_{2}$ is also marked to be restricted for chunk $n$.

After finding the inter-cell dominant interferer(s) to be restricted on each chunk and each UT, achievable rates $r_{m, n}^{(i)}$ are calculated. Now, the utility of chunk $n$ for UT $m$ can be expressed as:

$$
u_{m, n}^{(i)}=r_{m, n}^{(i)} d_{m}^{(i)}
$$

Utility matrix, $U_{M \times N}^{(i)}$, is formed with $u_{m, n}^{(i)}$, where each entry is associated with the corresponding interferer(s) to be restricted along with the achieved utility when the chunk is used by the respective UT.

2) Applying Hungarian algorithm and finding restriction requests: Hungarian algorithm is iteratively applied to $U_{M \times N}^{(i)}$ in order to prepare chunk restriction requests. Chunks are tentatively allocated (as the central controller might override the restriction requests) in order to reserve chunks for each UT with the following steps.
TABLE II

SECTOR-LEVEL ALGORITHM

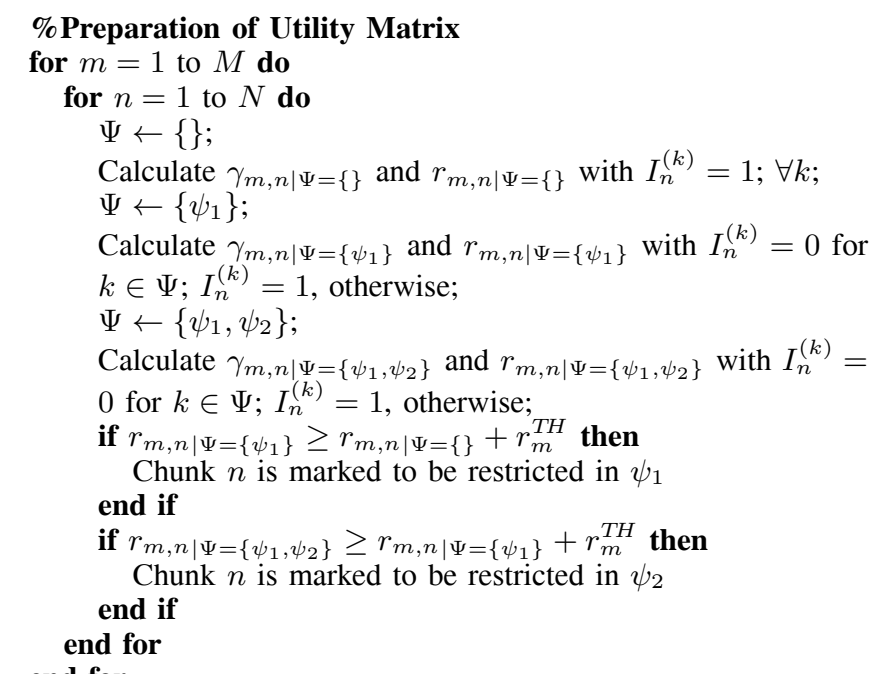

\section{end for}

Prepare utility matrix, $U_{M \times N}$, with $u_{m, n}=r_{m, n} \cdot d_{m}$ considering above restrictions

\section{\%Preparation of Chunk Restriction Requests}

Initialize $\mathcal{R}_{k}=\{\} ; \forall k$;

Initialize $\mathcal{U}=1 \cdots N$

$\hat{N} \leftarrow N$, where $\hat{N}$ is the number of unallocated chunks

while $\mathcal{U} \neq\{\}$ do

Apply Hungarian algorithm to $U_{M \times \hat{N}}$

if Allocated $\hat{n}^{t h}$ chunk has a mark for $k^{t h}$ sector interferer restriction then

Update corresponding entry of restriction request $\mathcal{R}_{k}$ end if

Remove columns of $U_{M \times \hat{N}}$ corresponding to allocated chunks

$N_{\text {alloc }}$

$\hat{N} \leftarrow \hat{N}-N_{\text {alloc }}$

end while

1) Apply Hungarian algorithm to $U_{M \times N}^{(i)}$. As $M<<N$, a maximum of $M$ chunks that yield the maximum sum utility will be allocated to the corresponding $M$ UTs.

2) If any of the $M$ chosen entries has restriction marked, the corresponding chunk will be placed in the restriction list for the corresponding interferer.

3) The columns belonging to the chosen entries are deleted from $U_{M \times N}^{(i)}$. The Hungarian algorithm is applied to the updated matrix.

4) Steps 2 and 3 are repeated until all chunks are tentatively allocated to UTs.

Now, each sector has a wish-list of chunks to be restricted for each of its neighboring sectors. These lists are forwarded to the central controller.

\section{B. Central-Level Algorithm}

The central controller receives requests from a cluster of BSs and resolves conflicting requests in an optimal manner. For a particular chunk, Fig. 5 shows an example problem to be solved at the central controller using its algorithm. In this figure, arrows with the solid and dashed lines indicate that interference received at the arrow-originating sector from the arrowhead sector is acceptable and unacceptable (requested 


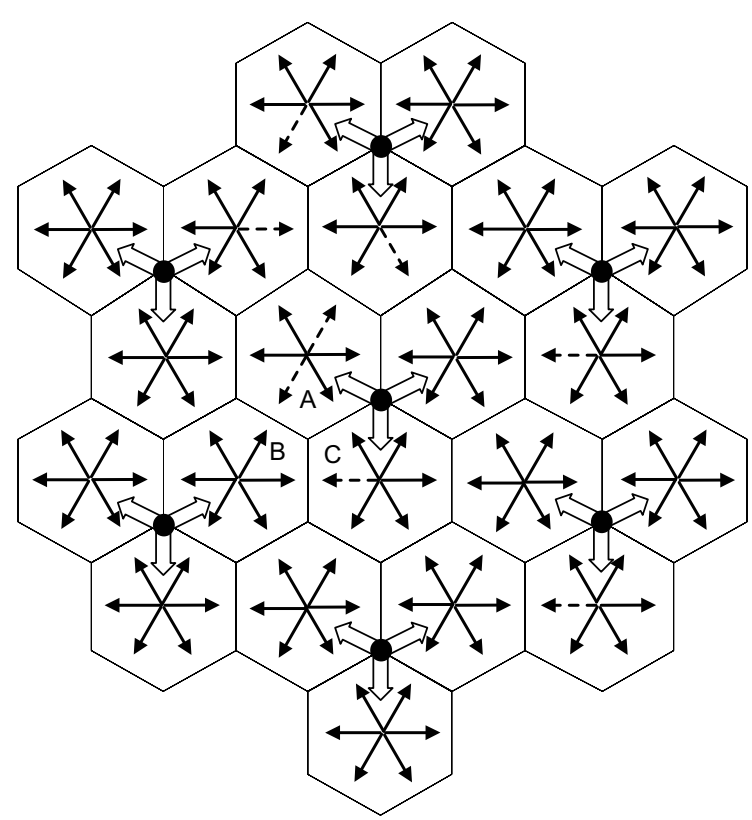

Fig. 5. Chunk restriction request for a particular chunk.

to be restricted), respectively. For example, for a chunk of interest, sector $B$ can tolerate interference from sector $A$, but the opposite is not true as there is a dashed arrow from sector $A$ toward sector $B$. In this case, either sector $A$ or $B$ has to be restricted for this chunk. For any chunk $n$, the problem at the central controller can be formulated formally as follows:

maximize

$$
Z=\sum_{i, j \in \Phi} u_{m_{i}, n}^{(i)}\left(1-x_{i, n}\right)+u_{m_{j}, n}^{(j)}\left(1-x_{j, n}\right),
$$

subject to

$$
x_{i, n}+x_{j, n} \leq 1,
$$

where $\Phi$ is the set of sectors in which each sector either requests or is requested for restriction to use chunk $n$; i.e., $i$ has restriction request to sector $j$ or vice versa or both have restriction requests to each other. UTs $m_{i}$ and $m_{j}$ are the candidates for chunk $n$ in sector $i$ and $j$, respectively. The variables $x_{i, n}$ and $x_{j, n}$ are binary which represent chunk restrictions. The value of $x_{i, n}$ (or $x_{j, n}$ ) is 1 if the chunk is restricted in sector $i$ (or $j$ ), otherwise, it is zero. A simple example with reference to Fig. 5 (considering only sectors $A, B$, and $C$ ) can be given as follows. Let us assume that for chunk $n$, sectors $A, B$, and $C$ attain utility of 4,6 , and 3 , respectively. In this case, the objective function is $Z=4\left(1-x_{A, n}\right)+6\left(1-x_{B, n}\right)+3\left(1-x_{C, n}\right)$, and the constraints are $x_{A, n}+x_{B, n} \leq 1$ and $x_{B, n}+x_{C, n} \leq 1$. Clearly, $x_{A, n}=0$, $x_{B, n}=1$, and $x_{C, n}=0$ provide maximized $Z$, and therefore, sector $B$ has to be restricted for chunk $n$.

The central controller resolves request conflicts and sends refined restriction lists to all involved BSs.

The proposed restriction processing can be done from time to time as long as the channel coherence time permits. This period is usually much longer than the scheduling interval and shorter than the channel coherence time. Once the chunk restriction list is available to a sector, the scheduler can perform chunk scheduling based on its own criteria. In that sense, the above chunk restriction technique can be considered as independent of scheduling. In this study, we use the iterative Hungarian algorithm and proportional fair scheduling in both the proposed and the reference schemes. Chunk restriction processing is performed on 6-chunk time-interval and scheduling is done at every chunk time duration.

It should be mentioned that the proposed scheme also works without the need for a central controller [16], however, with possible degradation in performance as conflict resolution is expected to be suboptimal. In such case, the scheme can take advantage of the inter-BS communications, for instance, using the X2 interface as defined in 3GPP LTE [30]. If two sectors wish to restrict a chunk to each other, the decision should result in favor of the sector that foresees higher utilization on that chunk. The pairwise comparison of utilization results in sub-optimal solution.

Currently, X2 interface supports information exchange between BSs (called evolved NodeB in LTE terminology) primarily for mobility management, load management, and interference coordination, which is controlled by X2AP protocol [31]. With necessary modifications in the protocol message, a BS can pass not only the indices of the chunks to be restricted but also the utilization seen on those chunks.

\section{Simulation Models and Parameters}

A total of 19 cell sites (i.e., 57 hexagonal sectors) are considered in the simulations, as shown in Fig. 2. The intersite distance is $1 \mathrm{~km}$. UTs are randomly distributed [32] in the centre 21 shaded sectors within a minimum and maximum radius in each sector. While the sector algorithm is executed in these 21 sectors, other sectors remain as interference contributors only. Performance statistics are collected from the 3 central sectors (i.e., BS1 as in Fig. 2).

The available spectrum of $45 \mathrm{MHz}$ in the $3.95 \mathrm{GHz}$ frequency band is available to each sector giving a total of 1152 sub-carriers each of which has a bandwidth of $39.0625 \mathrm{kHz}$. A chunk (sub-channel) consists of 8 consecutive sub-carriers. It is a time-frequency resource unit occupying $0.3456 \mathrm{~ms}$ and $312.5 \mathrm{KHz}$, which translates into $8 \times 12$ OFDM symbols [28].

All UTs are assumed to fall in the same service class. Both full-buffer and Poisson traffic models have been considered in this investigation. For the simplicity of simulations, a simple queuing model with Poisson arrival has been assumed for the downlink traffic. The arrival data rate and packet size are considered to be $5 \mathrm{Mbps}$ and 96 bytes, respectively.

Time-frequency-correlated Rayleigh channel samples are generated from the power delay profile for WINNER wide area scenario [32]. The user mobility is assumed to be 20 $\mathrm{km} / \mathrm{hr}$. The following exponential path-loss $(L)$ model has been used [32],

$$
\begin{array}{r}
L=\left(44.9-6.55 \log _{10}\left(h_{B S}\right)\right) \log _{10}(d)+34.46+ \\
5.83 \log _{10}\left(h_{B S}\right)+23 \log _{10}\left(f_{c} / 5.0\right)[\mathrm{dB}],
\end{array}
$$

where $h_{B S}$ and $d$ are the BS height and transmitter-receiver separation in meters, respectively, and $f_{c}$ is the carrier frequency in $\mathrm{GHz}$. 
The UT receive antennas are omni-directional, while the gain pattern for $120^{\circ}$ directional sector transmit antennas is considered to be as follows [28]:

$$
A(\theta)=-\min \left[12\left(\frac{\theta}{\theta_{3 d B}}\right)^{2}, 20\right],
$$

where the value of $\theta_{3 d B}$ is $70^{\circ}$ and $\theta$ varies from $-180^{\circ}$ to $180^{\circ}$. We have used a single-in-single-out (SISO) antenna configuration.

The average thermal noise power is calculated with a noise figure of $7 \mathrm{~dB}$. We have considered independent lognormal random variables with a standard deviation of $8 \mathrm{~dB}$ for shadowing. Sector transmit power is assumed to be 39.81 Watts and chunks are assigned powers so that the total used power is constant in all schemes. Retransmission for erroneous data is considered only in simulations with traffic model.

Adaptive modulation with a block low-density parity-check (B-LDPC) code is used. The thresholds for adaptive modulation and coding (AMC) modes are determined from a set of water-fall curves produced by the link level simulations [28] and considering 10\% block error rate (BLER). From binary phase shift keying (BPSK) to 64- quadrature amplitude modulation (64-QAM) have been used with varying code rates. Eight OFDM symbols (out of 96) distributed within each chunk are considered to be occupied by the reference signals and control signaling. Therefore, a chunk using quadrature PSK (QPSK) with a coding rate of $1 / 2$ can carry 88 information bits.

Note that for the full-buffer case, all available chunks are used in all sectors. Therefore, there is no interference uncertainty due to the unused residual resources. However, in the simulations with traffic model, it is possible that some resources may not be used in some sectors due to empty queues and/or poor channels which may result interference fluctuations; i.e., discrepancies between predicted and actual interference. An approach has been taken in order to minimize the effect of such fluctuations. Interference is estimated based on a probabilistic model. If a chunk is used at least once in the previous three scheduling instant in an interfering sector, then it is assumed that this particular interfering sector will use that particular chunk in the upcoming instant. This approach captures the dynamics of a multi-cell simulation more accurately than assuming a fixed allocation in the interfering cells. The statistics are collected based on actual SINR on the scheduled chunks. In this case, if the actual received SINR is less than the predicted one which is used at the transmitter to determine the AMC mode, then the packet is considered to be corrupted. Such retransmissions are scheduled in the next opportunity and given higher priority compared to the new packets.

End-to-end (E2E) delay performance is observed in the cases with traffic model. E2E delay is defined as the time difference between the packet arrival time at the transmitter queue and the successful reception of the last byte of the packet at the UT.

In order to solve the binary integer optimization problem at the central controller, YALMIP [33] and LPSOLVE (an integer linear programming (ILP) solver) [34] have been used along with MATLAB.

\section{Simulation Results}

Performance results have been observed for a user density of 12 UTs per sector. Performance of the proposed scheme is compared with that of a number of reference schemes such as reuse 1 , reuse 3 , and PFR schemes, where the reuse 1 represents a scheme with no coordination and the latter two are the examples of static interference coordination schemes. The reuse 1 scheme copes with the inter-cell interference and maximizes the utility in the presence of the worst-case interference. The reuse 3 and PFR schemes show benefits of static coordination over reuse 1 scheme. While all schemes achieve multiuser diversity, the gain in the proposed scheme is solely due to dynamic interference coordination.

We observe the CDF of the average UT throughput and compare the $5^{\text {th }}$ percentile throughput as a measure of the cell-edge UT performance. We also evaluate the CDF of E2E delay for the cases with traffic model. We used two different scheduling techniques such as the iterative Hungarian and proportional fair scheduling principles. Statistics are collected in the central 3 sectors and from 150 simulation drops, where each drop is simulated over a time duration of 100 chunks. In each drop, the throughput of the UT is averaged over this time duration.

The CDFs of UT throughput for the full-buffer case are shown in Fig. 6 and Fig. 7 when iterative Hungarian and proportional fair schedulers are used, respectively. The lower tail of the CDF is zoomed for the clarity to show the $5^{t h}$. percentile points. As expected, reuse 1 scheme performs very poorly with regard to the cell-edge throughput as there is no interference coordination. On the other hand, being a static interference coordination scheme, reuse 3 scheme provides enhanced cell-edge throughput, however, with a significant loss in sector throughput (i.e., around 54\%). The PFR scheme attains around 12 times gain in cell-edge throughput compared to the reuse 1 scheme, but with around $30 \%$ loss in sector throughput.

The proposed dynamic interference coordination scheme attains the most gain in cell-edge performance when the restricted chunks are not used at all. It is also observed that threshold criteria expressed in (6) provides a cell-edge throughput equivalent to that of the reuse 3 scheme while keeping sector throughput greater than that in PFR. Also, when restricted chunks are used with $10 \mathrm{~dB}$ lower power and the threshold is set to $r 2^{T H}$, proposed scheme achieves significantly higher cell-edge throughput without any impact on the sector throughput compared to the PFR scheme. Table III summarizes results for the full-buffer and with traffic model (in parenthesis), and compares cell-edge and sector throughputs for the reference and proposed schemes. Prop. 1 and Prop. 2 in the table refer to the scenarios when restricted chunks are not used and used with $10 \mathrm{~dB}$ lower power, respectively.

For simulations considering traffic model with Poisson arrival, the CDFs of average UT throughput for the iterative Hungarian and proportional fair schedulers have been illustrated in Fig. 8 and Fig. 9, respectively. Corresponding E2E delay performances are presented in Fig. 10 and Fig. 11, respectively. Similar to the full-buffer scenario, it is seen 
TABLE III

Cell-Edge vs Sector Throughput: Full-Buffer (Traffic Model, in Parenthesis)

\begin{tabular}{c|c|c|c|c|c|c}
\hline \hline & \multicolumn{3}{|c|}{ Iterative Hungarian } & \multicolumn{3}{c}{ Proportional Fair } \\
\hline Scheme & $\begin{array}{c}5^{t h} \text {-Perc. } \\
\text { in kbps }\end{array}$ & $\begin{array}{c}95^{t h}-\text { Perc. } \\
\text { in Mbps }\end{array}$ & $\begin{array}{c}\text { Sector TP } \\
\text { in Mbps }\end{array}$ & $\begin{array}{c}5^{t h} \text {-Perc. } \\
\text { in kbps }\end{array}$ & $\begin{array}{c}95^{t h} \text {-Perc. } \\
\text { in Mbps }\end{array}$ & $\begin{array}{c}\text { Sector TP } \\
\text { in Mbps }\end{array}$ \\
\hline \hline reuse 1 & $29.3(72.4)$ & $17.2(5.42)$ & $88.6(46.9)$ & $33.1(95.3)$ & $23.7(5.40)$ & $100.2(46.6)$ \\
reuse 3 & $795.1(805.5)$ & $5.2(4.89)$ & $42.0(41.3)$ & $674.3(661.0)$ & $6.2(5.27)$ & $46.0(42.5)$ \\
PFR & $347.4(392.8)$ & $11.8(5.35)$ & $64.3(45.6)$ & $386.4(461.3)$ & $16.3(5.40)$ & $72.5(46.6)$ \\
Prop. 1 with $r 1^{T H}$ & $486.8(405.5)$ & $12.6(5.40)$ & $77.8(48.1)$ & $586.9(424.8)$ & $15.8(5.40)$ & $84.7(47.4)$ \\
Prop. 2 with $r 1^{T H}$ & $310.9(317.7)$ & $17.5(5.41)$ & $88.6(47.8)$ & $391.5(357.1)$ & $21.9(5.40)$ & $99.0(47.1)$ \\
Prop. 1 with $r 2^{T H}$ & $750.3(711.9)$ & $11.3(5.35)$ & $73.1(47.2)$ & $773.4(605.4)$ & $13.9(5.37)$ & $79.5(46.3)$ \\
Prop. 2 with $r 2^{T H}$ & $464.7(449.6)$ & $17.2(5.39)$ & $88.0(47.4)$ & $520.1(472.3)$ & $21.8(5.39)$ & $98.4(46.3)$ \\
\hline
\end{tabular}

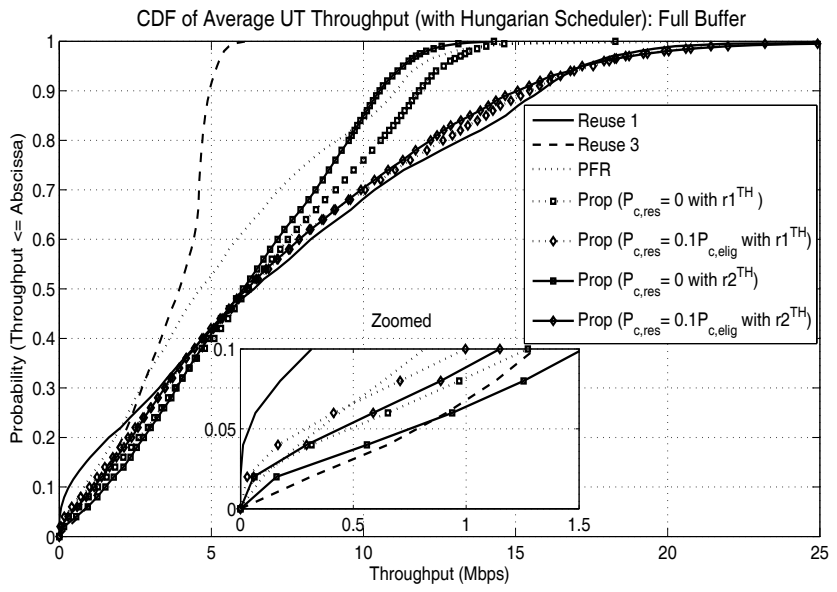

Fig. 6. CDF of UT throughput (Hungarian scheduler): full-buffer.

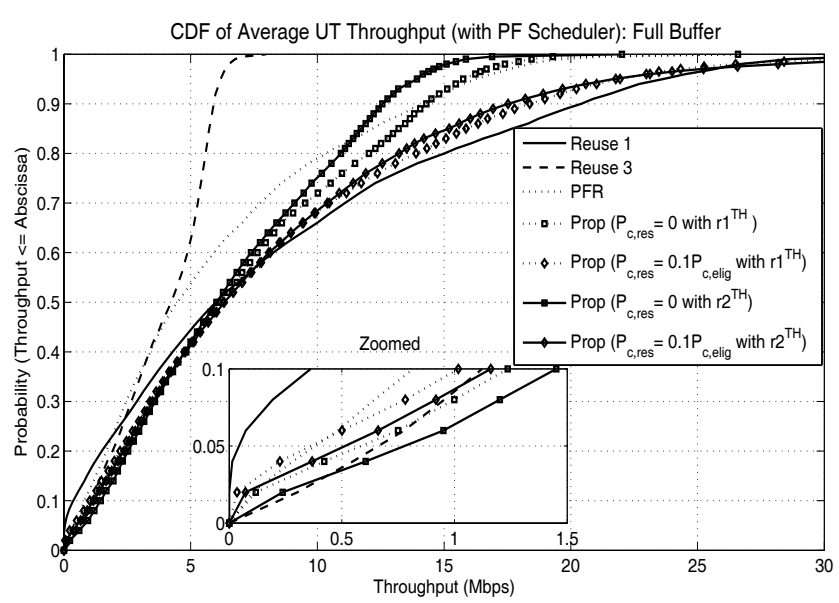

Fig. 7. CDF of UT throughput (PF scheduler): full-buffer.

that reuse 3 scheme achieves significant gain in cell-edge throughput, but with loss in sector throughput and degraded delay performance compared to the reuse 1 scheme. The performance of PFR falls between reuse 1 and reuse 3 schemes in terms of cell-edge and sector throughputs, and packet delay. Proposed scheme with $r 2^{T H}$ that does not use restricted chunks achieves cell-edge performance comparable to reuse 3 scheme while maintaining the sector throughput and delay performance comparable to reuse 1 scheme. It should be noted that the proposed schemes which use restricted chunks with $10 \mathrm{~dB}$ lower power do not bring any benefits in the cases with

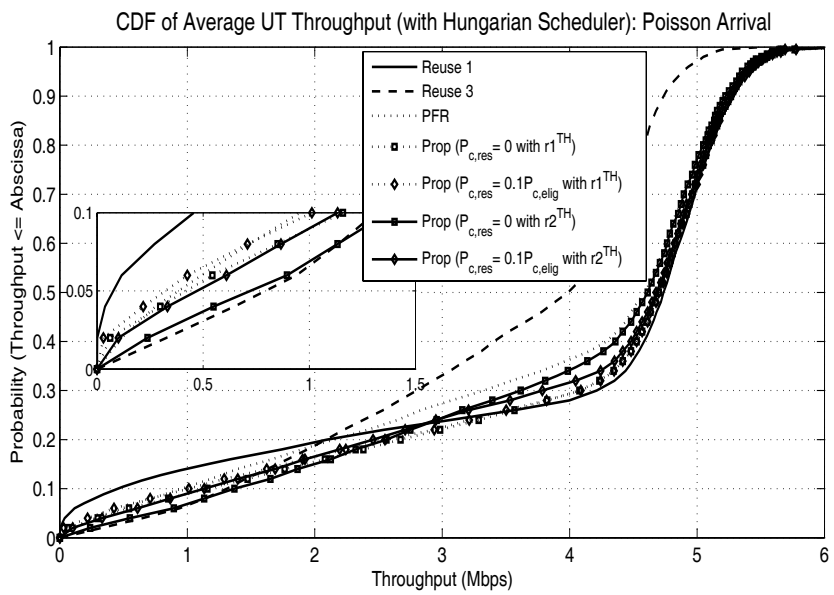

Fig. 8. CDF of UT throughput (Hungarian scheduler): Poisson arrival.

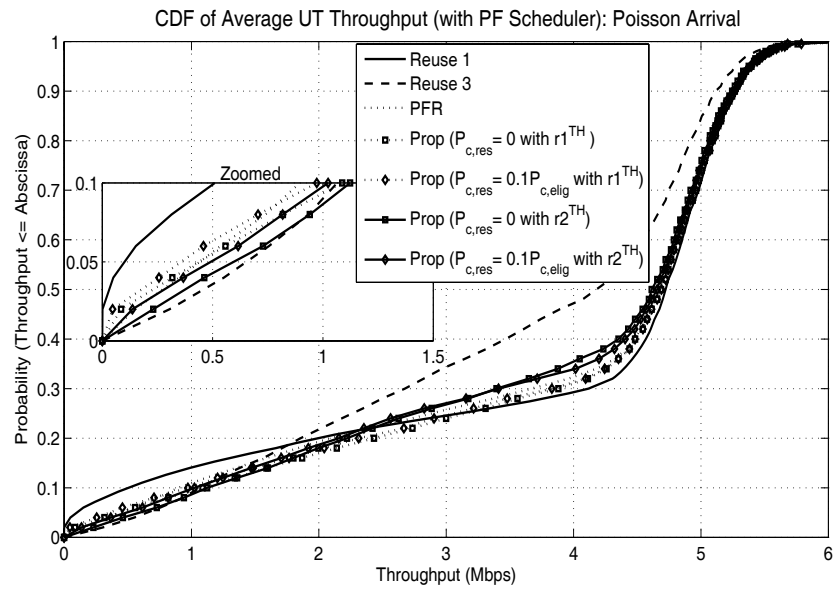

Fig. 9. CDF of UT throughput (PF scheduler): Poisson arrival.

a traffic model. The numbers in the parenthesis of Table III compares cell-edge and sector throughputs for all simulated schemes when a traffic model is used.

\section{IMPLEMENTATION COMPLEXITY ISSUES}

Two different kinds of complexities are associated with the proposed schemes; the computational complexities of the algorithms and the signaling overhead.

The overall complexity of the sector-level algorithm is dominated by the complexity of the Hungarian algorithm which is upper-bounded by $O\left(\min ^{2}(M, N) \cdot \max (M, N)\right)$ [35], where 


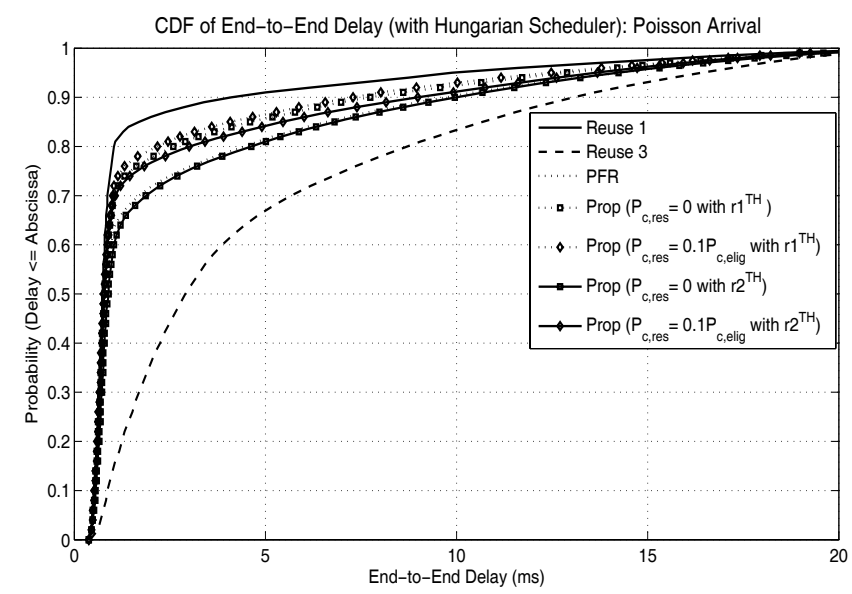

Fig. 10. CDF of E2E delay (Hungarian scheduler): Poisson arrival.

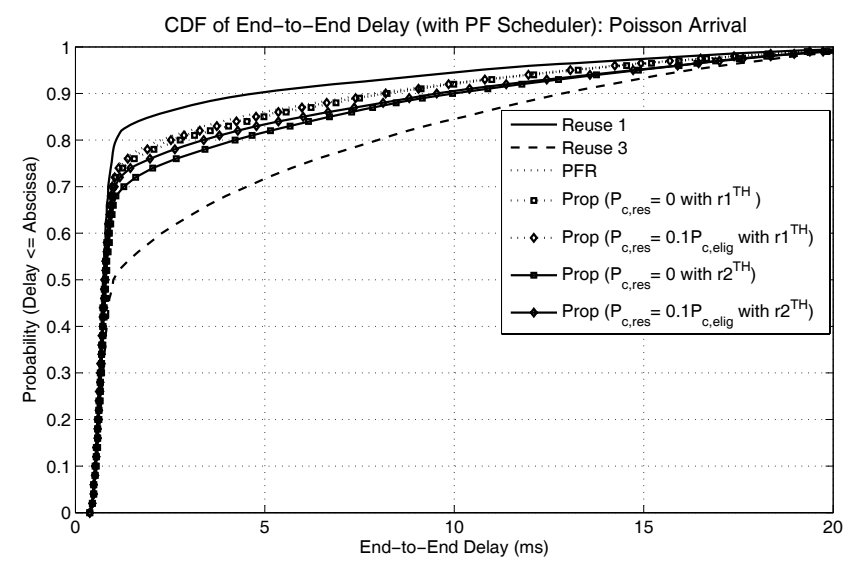

Fig. 11. CDF of E2E delay (PF scheduler): Poisson arrival.

$M$ and $N$ are the number of UTs and chunks, respectively. The complexity of the algorithm at the central controller depends on the number of sectors that have conflicting restriction requests. This determines the number of variables and constraints in the binary integer problem. For example, if there are 3 pair-wise conflicting requests for a particular chunk, then the number of binary variables to solve is 6 and the number of constrains is 9. Systems employing AMC are required to facilitate CSI feedback and are subjected to associated signaling overhead. In the proposed schemes that use threshold function (5), only rate deprived UTs are required to send information of two most dominant interference to the serving BS causing additional signaling overhead. All UTs in the proposed schemes with threshold function (6) are required to forward such information. Threshold functions in (5) and (6) can be considered as the trade-off between performance and overhead complexity. However, the rate of required overhead is related to the frequency of the channel reporting and resource allocation operations, which essentially depend on the mobility of the UTs and hence on the resulting channel coherence time. Therefore, a higher signaling overhead would have to be supported for higher mobility UTs. UTs in the reference static interference coordination schemes do not require to send any additional signaling other than the CSI.

In the proposed schemes, signaling between BS and the central entity is also required which can be performed using high data rate backbone connections such as fiber links and thus it is less of an issue.

\section{CONCLUSiOnS}

A novel viable interference coordination scheme using downlink multi-cell chunk allocation with dynamic inter-cell coordination is presented in this paper. The performance of the proposed schemes is compared with that of a number of static coordination-based reference schemes available in the literature. It is observed from the simulation results that the static coordination schemes achieve enhanced cell-edge throughput only with a significant loss in sector throughput. On the other hand, the proposed schemes achieve equivalent or better cell-edge throughput without impacting the sector throughput. All simulated schemes achieve multi-user diversity through the schedulers. Therefore, the observed performance gain in the proposed scheme is solely due to dynamic interference coordination. Enhanced cell edge throughput in the proposed scheme can potentially allow a smaller number of BSs to cover a region yielding substantial savings in the deployment cost. As it does not require any frequency planning, the proposed scheme is not only effective for macrocell environment, it can be applied to future femtocell BSs where user terminals are expected to experience severe interference from neighboring macrocell BSs.

Although we have used a central entity for the resolution of the conflicting requests, the algorithm can be applied to radio access networks (RANs) without a central controller, for example, 3GPP LTE and LTE-Advanced networks. In this case, resolutions can be performed among neighboring sectors through negotiations using X2 interface that inter-connects BSs.

In the sector algorithm, a simple utility function has been used, where the considered utility is proportional to the achieved rate on the chunk of interest and UT resource demand at the allocation instant. However, it may be possible to devise a more comprehensive utility function that may consider other critical factors necessary for improved network performance. We, nevertheless, show the potential of a dynamic interference avoidance scheme and highlight its trade-off between the performance and complexity adjusted through the tunable threshold function.

\section{REFERENCES}

[1] Part 11: Wireless LAN Medium Access Control (MAC) and Physical Layer (PHY) Specifications, IEEE Standard 802.11-2007, Dec. 2007. [Online]. Available: http://standards.ieee.org.

[2] "Mobile WiMAX_part I: a technical overview and performance evaluation," White Paper, WiMAX Forum, Aug. 2006.

[3] E-UTRA and E-UTRAN Overall description; Stage 2 (Release 8), 3GPP Technical Specification TS 36.300 V8.7.0, Dec. 2008. [Online]. Available: http://www.3gpp.org.

[4] Requirements for Further Advancements for E-UTRA (LTE-Advanced) (Release 8), 3GPP Technical Specification TR 36.913 V8.0.0, Jun. 2008. [Online]. Available: http://www.3gpp.org.

[5] WINNER II System Concepts Description, WINNER II Deliverable D6.13.14, Jan. 2008. [Online]. Available: http://www.ist-winner.org.

[6] Initial Report on Advanced Multiple Antenna Systems, WINNER+ Deliverable D1.4, Jan. 2009. [Online]. Available: http://projects.celticinitiative.org/winner+.

[7] Interference Avoidance Concepts, WINNER II Deliverable D4.7.2, Jun. 2007. [Online]. Available: http://www.ist-winner.org. 
[8] Physical Layer Aspects for Evolved Universal Terrestrial Radio Access (UTRA) (Release 7), 3GPP Technical Report TR 25.814 V7.1.0, Sep. 2006. [Online]. Available: http://www.3gpp.org.

[9] M. Rahman and H. Yanikomeroglu, "Interference avoidance through dynamic downlink OFDMA subchannel allocation using intercell coordination," in Proc. IEEE Veh. Technol. Conf. (VTC2008-Spring), May 2008, pp. $1630-1635$.

[10] V. H. MacDonald, "The cellular concept," Bell Syst. Tech. J., vol. 58, pp. 15-41, Jan. 1979.

[11] OFDMA Downlink Inter-Cell Interference Mitigation, 3GPP Project Document R1-060291, Feb. 2006. [Online]. Available: http://www.3gpp.org.

[12] Soft Frequency Reuse Scheme for UTRAN LTE, 3GPP Project Document R1-050 507, May 2005. [Online]. Available: http://www.3gpp.org.

[13] V. Chandrasekhar, J. G. Andrews, and A. Gatherer, "Femtocell networks: a survey," IEEE Commun. Mag., pp. 59-67, Sept. 2008.

[14] Further Discussion on Adaptive Fractional Frequency Reuse, 3GPP Project Document R1-072762, Jun. 2007. [Online]. Available: http://www.3gpp.org.

[15] S. H. Ali and V. C. M. Leung, "Dynamic frequency allocation in fractional frequency reused OFDMA networks," IEEE Trans. Wireless Commun., vol. 8, pp. 4286-4295, Aug. 2009.

[16] M. Rahman, H. Yanikomeroglu, and W. Wong, "Interference avoidance with dynamic inter-cell coordination for downlink LTE system," in Proc. IEEE Wireless Commun. Netw. Conf. (WCNC), Apr. 2009.

[17] M. C. Necker, "Scheduling constraints and interference graph properties for graph-based interference coordination in cellular OFDMA networks," Springer J. Mobile Netw. Appl., vol. 14, pp. 539-550, Jan. 2009.

[18] R. Y. Chang, Z. Tao, J. Zhang, and C. C. J. Kuo, "Multicell OFDMA downlink resource allocation using a graphic framework," IEEE Trans. Veh. Technol., vol. 58, pp. 3494-3507, Sept. 2009.

[19] C. Y. Wong, R. S. Cheng, K. B. Letaief, and R. D. Murch, "Multiuser OFDM with adaptive subcarrier, bit, and power allocation," IEEE J. Sel. Areas Commun., vol. 17, pp. 1747-1757, Oct. 1999.

[20] W. Rhee and J. M. Cioffi, "Increase in capacity of multiuser OFDM system using dynamic subchannel allocation," in Proc. IEEE Veh. Technol. Conf. (VTC2000-Spring), May 2000, pp. 1085-1089.

[21] D. Niyato and E. Hossain, "Adaptive fair subcarrier/rate allocation in multirate OFDMA networks: radio link level queuing performance analysis," IEEE Trans. Veh. Technol., vol. 55, pp. 1897-1907, Nov. 2006.

[22] H. Kim, Y. Han, and J. Koo, "Optimal subchannel allocation scheme in multicell OFDMA systems," in Proc. IEEE Veh. Technol. Conf. (VTC2004-Spring), May 2004, pp. 1821-1825.

[23] G. Li and H. Liu, "Downlink dynamic resource allocation for multi-cell OFDMA systems," in Proc. IEEE Veh. Technol. Conf. (VTC2003-Fall), Oct. 2003, pp. 1698-1702.

[24] M. Garey and D. S. Johnson, Computers and Intractability: A Guide to the Theory of NP-Completeness. W.H. Freeman and Company, 1979.

[25] Further Advancements for E-UTRA Physical Layer Aspects (Release 9), 3GPP Technical Specification TR 36.814 V0.4.1, Feb. 2009. [Online]. Available: http://www.3gpp.org.

[26] Further Analysis of Soft Frequency Reuse Scheme, 3GPP Project Document R1-050841, Sept. 2005. [Online]. Available: http://www.3gpp.org.

[27] M. Sternad, T. Ottosson, A. Ahlen, and A. Svensson, "Attaining both coverage and high spectral efficiency with adaptive OFDM downlinks," in Proc. IEEE Veh. Technol. Conf. (VTC2003-Fall), Oct. 2003, pp. 24862490.

[28] Test Scenarios and Calibration Cases Issue 2, WINNER II Deliverable D6.13.7, Dec. 2006. [Online]. Available: http://www.ist-winner.org.

[29] H. W. Kuhn, "The Hungarian method for the assignment problem," Naval Research Logistics Quarterly, vol. 2, pp. 83-97, 1955.

[30] X2 General Aspects and Principles (Release 8), 3GPP Technical Specifications TS 36.420, Dec. 2008. [Online]. Available: http://www.3gpp.org.

[31] X2 Application Protocol (X2AP) (Release 8), 3GPP Technical Specifications TS 36.423, June 2009. [Online]. Available: http://www.3gpp.org.
[32] WINNER II Channel Models, WINNER II Deliverable D1.1.2 V1.2, Sep. 2007. [Online]. Available: http://www.ist-winner.org.

[33] J. Löfberg, "YALMIP: a toolbox for modeling and optimization in MATLAB," in Proc. IEEE International Conf. Computer-Aided Control Syst. Design, Taipei, Taiwan, 2004.

[34] M. Berkelaar, K. Eikland, and P. Notebaert (2009), lp_solve version 5.5. [Online]. Available: http://lpsolve.sourceforge.net/5.5.

[35] F. Burgeios and J. C. Lassalle, "An extension of Munkres algorithm for the assignment problem to rectangular matrices," Commun. ACM, vol. 14, no. 12, pp. 802-804, 1971.

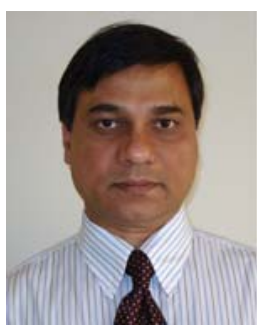

Mahmudur Rahman received his Bachelor of Science degree in Electrical \& Electronic Engineering from Bangladesh University of Engineering \& Technology (BUET), Dhaka, Bangladesh, in 1991. $\mathrm{He}$ obtained a Master of Engineering degree in Telecommunications from Asian Institute of Technology (AIT), Bangkok, Thailand and a Master of Applied Science degree in Electrical Engineering from Carleton University, Ottawa, Canada, in 1994 and 2004, respectively.

Mr. Rahman worked for Atomic Energy Commission (Bangladesh), Johnson Electric Industrial Ltd. (Thailand), and ACE Electronics Industries Co., Ltd. (Thailand) at different times of his career. He is currently pursuing a Ph.D. degree in Systems and Computer Engineering at Carleton University, Ottawa, Canada, and working as a Research Engineer in Communications Research Centre (CRC), Ottawa, Canada. His current research interests include radio resource management, multi-hop wireless networks, inter-cell coordination, interference management, and cognitive radio systems.

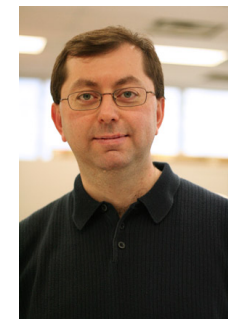

Halim Yanikomeroglu received a B.Sc. degree in Electrical and Electronics Engineering from the Middle East Technical University, Ankara, Turkey, in 1990, and a M.A.Sc. degree in Electrical Engineering (now ECE) and a Ph.D. degree in Electrical and Computer Engineering from the University of Toronto, Canada, in 1992 and 1998, respectively. He was with the R\&D Group of Marconi Kominikasyon A.S., Ankara, Turkey, from 1993 to 1994.

Since 1998 Dr. Yanikomeroglu has been with the Department of Systems and Computer Engineering at Carleton University, Ottawa, where he is now an Associate Professor with tenure. Dr. Yanikomeroglu's research interests cover many aspects of the physical, medium access, and networking layers of wireless communications with a special emphasis on multihop/relay/mesh networks and cooperative communications. Dr. Yanikomeroglu's research is currently funded by Huawei (China), RIM (Canada), Communications Research Centre of Canada (CRC), Nortel, and NSERC. Dr. Yanikomeroglu is a recipient of the Carleton University Research Achievement Award 2009.

Dr. Yanikomeroglu has been involved in the steering committees and technical program committees of numerous international conferences; he has also given 18 tutorials in such conferences. Dr. Yanikomeroglu is a member of the Steering Committee of the IEEE Wireless Communications and Networking Conference (WCNC), and has been involved in the organization of this conference over the years, including serving as the Technical Program Co-Chair of WCNC 2004 and the Technical Program Chair of WCNC 2008. Dr. Yanikomeroglu is the General Co-Chair of the IEEE Vehicular Technology Conference to be held in Ottawa in September 2010 (VTC2010-Fall).

Dr. Yanikomeroglu was an editor for IEEE TRANS ACTIONS ON WIRELESS Communications [2002-2005] and IEEE Communications SuRveys \& TUTORIALS [2002-2003], and a guest editor for Wiley JOURNAL ON Wireless COMMUNiCATIONS \& MoBile Computing. He was an Officer of IEEE's Technical Committee on Personal Communications (Chair: 200506, Vice-Chair: 2003-04, Secretary: 2001-02), and he was also a member of the IEEE Communications Society's Technical Activities Council (2005-06).

Dr. Yanikomeroglu is an Adjunct Professor at Prince Sultan Advanced Technologies and Research Institute (PSATRI) at King Saud University, Riyadh, Saudi Arabia; he is also a registered Professional Engineer in the province of Ontario, Canada. 